\title{
Downregulation of GLUT3 impairs STYK1/NOK-mediated metabolic reprogramming and proliferation in NIH-3T3 cells
}

\author{
WEIYE SHI, YU FU and YINGZE WANG \\ Cell Engineering Laboratory, College of Biological Science and Engineering, \\ Hebei University of Science and Technology, Shijiazhuang, Hebei 050018, P.R. China
}

Received February 3, 2021; Accepted April 30, 2021

DOI: $10.3892 / \mathrm{ol} .2021 .12788$

\begin{abstract}
Serine threonine tyrosine kinase 1 (STYK1)/novel oncogene with kinase domain (NOK) has been demonstrated to promote cell carcinogenesis and tumorigenesis, as well as to strengthen cellular aerobic glycolysis, which is considered to be a defining hallmark of cancer. As the carriers of glucose into cells, glucose transporters (GLUTs) are important participants in cellular glucose metabolism and even tumorigenesis. However, to the best of our knowledge, the role of GLUTs in biological events caused by STYK1/NOK has not yet been reported. The present study assessed GLUT3 as a key transporter, and glucose consumption and lactate production assays revealed that downregulation of GLUT3 impaired STYK1/NOK-induced augmented glucose uptake and lactate production, and RT-qPCR and western blotting confirmed that GLUT3 knockdown attenuated the STYK1/NOK-induced increase in the expression levels of key enzymes implicated in glycolysis. Furthermore, MTT and Transwell assays demonstrated that STYK1/NOK-triggered cell proliferation and migration were also markedly decreased following knockdown of GLUT3. To the best of our knowledge, the present study is the first to demonstrate that GLUT3 serves a prominent role in STYK1/NOK-driven aerobic glycolysis and cell proliferation characteristics. These findings may provide a clue for the investigation of the oncogenic activity of STYK1/NOK and for the identification of potential tumor therapy targets associated with GLUT3.
\end{abstract}

\section{Introduction}

Serine threonine tyrosine kinase 1 (STYK1), also known as novel oncogene with kinase domain (NOK), belongs to the

Correspondence to: Dr Weiye Shi, Cell Engineering Laboratory, College of Biological Science and Engineering, Hebei University of Science and Technology, 26 Yuxiang Street, Shijiazhuang, Hebei 050018, P.R. China

E-mail:916153884@qq.com

Key words: glucose transporter 3, serine threonine tyrosine kinase $1 /$ novel oncogene with kinase domain, aerobic glycolysis, cell proliferation, cell migration receptor protein tyrosine kinases (RPTKs) subfamily (1); it has been demonstrated to be a potent oncogene that enhances cell proliferation in vitro, and drives both tumorigenesis and metastasis in animal model systems (2). Aberrant STYK1/NOK expression has been identified in a wide range of cancer types, including lung, ovarian, breast, colorectal, prostate and renal cell cancer (3-8). Notably, cells overexpressing STYK1/NOK exhibit a similar metabolic profile compared with cancer cells, namely functions in aerobic glycolysis or the Warburg effect, which is reflected in augmented glucose uptake and lactate production, upregulation of key glycolytic enzymes and regulators, impaired electron transport and mitochondrial oxidative phosphorylation (OXPHOS) (9).

As a consequence of aerobic glycolysis, cancer cells become heavily dependent on both glycolysis and glucose uptake. In order to incorporate sufficient amounts of glucose, cells increase the expression levels of different glucose transporters (GLUTs). At present, 14 types of human GLUTs encoded by different genes have been identified. Although their substrate specificity and tissue distribution are different, these GLUTs have common sequence characteristics and are highly conserved in numerous species, such as mice and rats (10). According to the differences in extracellular structure, these GLUTs can be classified into three categories: Class I (GLUT1-4), class II (GLUT5, 7, 9 and 11) and class III (GLUT6, 8, 10, 12 and 13) (11). Class I GLUTs were discovered first and studied in depth. Among them, GLUT1 and GLUT3 are widely distributed in the plasma membrane of all tissues and cells, and are responsible for maintaining the basic level of glucose uptake under normal physiological conditions (10-12). GLUT2 is mainly present in certain tissues with high glucose concentrations, such as those in the intestine and liver (13). GLUT4 is highly expressed in insulin-sensitive tissues, including brown and white fat, skeletal muscle and the myocardium (10). The newly discovered GLUT14 has 95\% sequence homology with GLUT3 and is only present in the testis; its role in glucose transport remains unclear.

Dysfunctions of certain GLUTs are closely associated with various diseases. Accumulating data have indicated that most tumor tissues have an abnormal GLUT expression profile compared with normal tissues, which is crucial for maintaining the proliferation, metastasis and survival of cancer cells under hypoxia $(14,15)$. In recent years, an increasing number of researchers have paid attention to the structural 
characteristics, the expression and regulation, and the clinical application of the main GLUTs in terms of their role as malignant tumor markers (16-18). However, most reports have focused on a specific type of tumor cell, and there are relatively few studies on the role of GLUTs in carcinogenic RPTKs, including STYK1/NOK-mediated malignant transformation and tumorigenesis (19-21).

The present study focused on the most significant class I GLUTs (GLUT1-4) and provided evidence for the functional involvement of the GLUT3 transporter in STYK1/NOK-mediated metabolic reprogramming and cell proliferation characteristics.

\section{Materials and methods}

Cell lines and reagents. The murine NIH-3T3 fibroblast cell line was obtained from the China Infrastructure of Cell Line Resources, Institute of Basic Medical Sciences, Chinese Academy of Medical Sciences. NIH-3T3 cells were grown in DMEM supplemented with $10 \%$ FBS (both Thermo Fisher Scientific, Inc.) at $37^{\circ} \mathrm{C}$ with $5 \% \quad \mathrm{CO}_{2}$. The following antibodies were used in the present study: Anti-STYK1 (cat. no. 18028-1-AP), anti-GLUT1 (cat. no. 21829-1-AP), anti-GLUT2 (cat. no. 20436-1-AP), anti-GLUT4 (cat. no. 21048-1-AP), anti-hexokinase (HK)1 (cat. no. 19662-1-AP), anti-platelet phosphofructokinase (PFKP) (cat. no. 13389-1-AP) and anti-pyruvate kinase (PKM)1 (cat. no. 15821-1-AP), all from ProteinTech Group, Inc.; anti-GLUT3 (cat. no. ab191071) and anti-pyruvate dehydrogenase $\alpha 1$ (PDHA1) (cat. no. ab168379), all from Abcam, Inc.; anti- $\beta$-actin (cat. no. 4970) and anti- $\beta$-tubulin (cat. no. 2146), both from Cell Signaling Technology, Inc.; and HRP-conjugated secondary antibody (cat. no. TA130003) from OriGene Technologies, Inc. MTT, DMSO and G418 were purchased from Sigma-Aldrich; Merck KGaA.

Plasmid construction and transient transfection. The pcDNA3.0 and pcDNA3.0-STYK1/NOK plasmids were constructed previously (9). For construction of pSilencer-small interfering RNA (si/siRNA) GLUT3, the single-stranded oligonucleotides (5'-AGCTTAAGTAGC TAAGTCGGTTGAAACTCGAGTTTCAACCGACTTAGC TACTTG-3' and 5'-GATCCAAGTAGCTAAGTCGGTTGA AACTCGAGTTTCAACCGACTTAGCTACTTA-3') were annealed to double strands before being subcloned into the HindIII and BamHI sites of pSilencer 4.1-CMV neo to form the pSilencer-siGLUT3 construct. For construction of pSilencer-small interfering RNA (si/siRNA) control, the single-stranded oligonucleotides (5'-AGCTTAATGGAT CAATGGCTGGTAAACTCGAGTTTACCAGCCATTGAT CCATTG-3' and 5'-GATCCAATGGATCAATGGCTGGTA AACTCGAGTTTACCAGCCATTGATCCATTA-3') were annealed to double strands before being subcloned into the HindIII and BamHI sites of pSilencer 4.1-CMV neo to form the pSilencer-siCtrl construct. All enzymes and reagents used for plasmid construction were purchased from Ambion, Inc. For transient transfection, NIH-3T3 cells at $\sim 80 \%$ confluence were transiently transfected with $4 \mu \mathrm{g}$ plasmid DNA using Lipofectamine ${ }^{\circledR} 2000$ transfection reagent (Thermo Fisher Scientific, Inc.) according to the manufacturer's protocol.
Construction of the NIH-3T3 stable cells. Plasmids pcDNA3.0 or pcDNA3.0-STYK1/NOK $(4 \mu \mathrm{g})$ were transfected into NIH-3T3 cells. After $24 \mathrm{~h}$ of transfection, the cell culture medium was replaced with fresh medium containing $600 \mu \mathrm{g} / \mathrm{ml} \mathrm{G} 418$. After 2 weeks of screening, cell colonies were picked up and expanded in a 24-well tissue culture plate. Finally, reverse transcription-quantitative PCR (RT-qPCR) and western blotting were performed to detect STYK1/NOK expression. NIH-3T3-pcDNA3.0 stable cells were represented as 'vehicle' and NIH-3T3-pcDNA3.0-STYK1/NOK stable cells were represented as 'STYK1/NOK'.

$R T-P C R$ and $R T-q P C R$. Total RNA was extracted from the cultured cells using TRIzol ${ }^{\circledR}$ (Invitrogen; Thermo Fisher Scientific, Inc.). RT-PCR was performed using the PrimeScript one-step RT-PCR kit (Takara Biotechnology Co., Ltd.) according to the manufacturer's protocols. The operating conditions for RT-PCR were as follows: $50^{\circ} \mathrm{C}$ for $35 \mathrm{~min}$ for reverse transcription and $94^{\circ} \mathrm{C}$ for $5 \mathrm{~min}$ for denaturation. The PCR conditions were: $94^{\circ} \mathrm{C}$ for $30 \mathrm{sec}, 50^{\circ} \mathrm{C}$ for $30 \mathrm{sec}$ and $72^{\circ} \mathrm{C}$ for $50 \mathrm{sec}$, repeated for $20-30$ cycles; the reaction was extended at $72^{\circ} \mathrm{C}$ for $10 \mathrm{~min}$ before the reaction product was stored at $4^{\circ} \mathrm{C}$. RT-qPCR was performed using the One Step SYBR PrimeScript RT-PCR kit II (Perfect Real Time; Takara Biotechnology Co., Ltd.). The operating conditions for RT-qPCR were: $42^{\circ} \mathrm{C}$ for $5 \mathrm{~min}$ and $95^{\circ} \mathrm{C}$ for $10 \mathrm{sec} ; 95^{\circ} \mathrm{C}$ for $5 \mathrm{sec}$ and $60^{\circ} \mathrm{C}$ for $30 \mathrm{sec}$ (repeated for 40 cycles). The dissociation of the reaction products was from $55^{\circ} \mathrm{C}$ to $95^{\circ} \mathrm{C}$ as the temperature increased at a rate of $0.2^{\circ} \mathrm{C}$ per $10 \mathrm{sec}$. The gene expression levels of $\beta$-actin were used as an internal control. The RT-qPCR results were calculated using the $2^{-\Delta \Delta C q}$ method (22). All primers were designed using the PrimerBank online program (https://pga.mgh.harvard.edu/primerbank/). Primer sequences are listed in Table I.

Western blot analysis. Western blotting was performed as described previously (23). Cells were collected and lysed in RIPA buffer (Beijing Solarbio Science \& Technology Co., Ltd.) containing 0.1 M PMSF, protease and phosphatase inhibitor cocktail for $30 \mathrm{~min}$ on ice. The lysates were centrifuged at $12,000 \mathrm{x} \mathrm{g}$ at $4^{\circ} \mathrm{C}$ for $15 \mathrm{~min}$, and the supernatant was collected. Protein concentration was determined by the BCA method. Cell lysates $(20 \mu \mathrm{g})$ were separated using a $10 \%$ gel by SDS-PAGE and transferred to a nitrocellulose membrane (Cytiva) at $200 \mathrm{~mA}$ for $1.5 \mathrm{~h}$. The membrane was blocked with 5\% BSA (Beijing Solarbio Science \& Technology Co., Ltd.) for $1 \mathrm{~h}$ at room temperature, probed with a primary antibody (dilution, $1: 1,000$ ) at $4^{\circ} \mathrm{C}$ overnight and an appropriate secondary antibody (dilution, 1:5,000) for $1 \mathrm{~h}$ at room temperature. Finally, the proteins were visualized using EasySee Western Blot kit (Beijing TransGen Biotech Co., Ltd., Beijing, China), and imaged and quantified using ChemiDoc MP Imaging system (Image Lab Software, version 4.1; Bio-Rad Laboratories Co., Ltd.).

Glucose consumption and lactate production assays. For the glucose consumption assay, cells were seeded into a 24-well culture plate in DMEM supplemented with $10 \%$ FBS. After $48 \mathrm{~h}, 100 \mu \mathrm{l}$ culture medium was obtained from each well for the determination of glucose content using a glucose assay 
Table I. Primers used in RT-PCR/RT-qPCR analysis.

\begin{tabular}{|c|c|c|c|c|}
\hline Gene name & GenBank ID & Forward primer $\left(5^{\prime}-3^{\prime}\right)$ & Reverse primer $\left(5^{\prime}-3^{\prime}\right)$ & $\begin{array}{c}\text { Size of } \\
\text { product, bp }\end{array}$ \\
\hline$\beta$-actin & BC009275 & CAGCCTCGTCCCGTAGACA & CGCTCCTGGAAGATGGTGAT & 161 \\
\hline STYK1/NOK & KP729000 & TCACCTAGAGAGCTGCGCTT & CGTAGTCTGGGACGTCGTATG & 181 \\
\hline GLUT1 & NM_011400 & GATCCCAGCAGCAAGAAGGT & AGAGACCAAAGCGTGGTGAG & 197 \\
\hline GLUT2 & NM_031197 & TGAGTTCCTTCCAGTTCGGC & TGTAAGTGGGGTGTCTGTGC & 152 \\
\hline GLUT3 & NM_011401 & CAGGAATCTTCAAGGACGCGG & CGAAATCGTCATGAAAACGGAGC & 179 \\
\hline GLUT4 & NM_009204 & ATTCTGCTGCCCTTCTGTCC & GGAGCTGGAGCAAGGACATT & 184 \\
\hline HK1 & NM_001146100 & GAGTCTGAGGTCTACGACACC & CCCACGGGTAATTTCTTGTCC & 131 \\
\hline PFKP & NM_019703 & CGCCTATCCGAAGTACCTGGA & CCCCGTGTAGATTCCCATGC & 130 \\
\hline PKM & NM_011099 & GCCGCCTGGACATTGACTC & CCATGAGAGAAATTCAGCCGAG & 145 \\
\hline
\end{tabular}

STYK1/NOK, serine threonine tyrosine kinase 1/novel oncogene with kinase-domain; GLUT, glucose transporter; HK1, hexokinase 1; PFKP, platelet phosphofructokinase; PKM, pyruvate kinase.

kit (cat. no. BC2490; Beijing Solarbio Science \& Technology Co., Ltd.) according to the manufacturer's instructions. For the lactate production assay, cells were seeded into a 6 -well culture plate in normal growth medium. After $48 \mathrm{~h}, 1 \times 10^{6}$ cells were collected from each well for the determination of lactate content using a lactate assay kit (cat. no. BC2230; Beijing Solarbio Science \& Technology Co., Ltd.) according to the manufacturer's instructions.

Cell proliferation assay using MTT. For the proliferation assay, cells were seeded into 96 -well plates at a density of 5,000 cells per well. After incubation for 24, 48 and $72 \mathrm{~h}$, cell proliferation was assessed by the addition of MTT at a final concentration of $0.5 \mathrm{mg} / \mathrm{ml}$ for $1-4 \mathrm{~h}$. After removing the culture medium, the cells in each well were re-suspended with $150 \mu \mathrm{l} \mathrm{DMSO}$ and then shaken for $10 \mathrm{~min}$ at $37^{\circ} \mathrm{C}$ to fully dissolve the crystals. The reaction products were measured at an optical density of $490 \mathrm{~nm}$ with a spectrophotometer.

Cell migration assay. Cell migration was analyzed using 8.0- $\mu \mathrm{m}$ Transwell ${ }^{\circledR}$ Inserts (Corning Life Sciences). Briefly, $1 \times 10^{5}$ cells were seeded into the upper chamber supplemented with $100 \mu \mathrm{l}$ serum-free medium, while $650 \mu 1$ DMEM supplemented with $10 \%$ FBS was added to the lower chamber as the inducer. Following $24 \mathrm{~h}$ of incubation with $5 \% \mathrm{CO}_{2}$ at $37^{\circ} \mathrm{C}$, non-migrated cells in the upper chamber were removed using a cotton swab, while migrated cells in the lower chamber were stained with $0.1 \%$ crystal violet for $10 \mathrm{~min}$ at room temperature. Finally, the cells were washed with PBS and counted under an inverted fluorescence microscope (magnification, x200; Olympus Corporation) using the image processing program ImageJ_v1.8.0 software (National Institutes of Health). Images of five random fields of view were captured for each group for analysis.

Statistical analysis. All statistical analyses were performed using GraphPad Prism 8 software (GraphPad Software Inc.). Student's t-test or one-way analysis of variance (ANOVA) followed by Tukey's post-hoc test was conducted to account for the comparison of two groups and multiple groups, respectively. Data are presented as the mean $\pm \mathrm{SD}$. $\mathrm{P}<0.05$ was considered to indicate a statistically significant difference.

\section{Results}

Effects of STYK1/NOK on the expression of GLUT1-4 participating in the glycolysis process. As aforementioned, GLUTs are required for glucose to enter cells on most occasions. Class I GLUTs (GLUT1-4) were discovered first and may serve an important role in this process. Our previous study revealed that STYK1/NOK can promote glucose uptake in NIH-3T3 cells (9), which is likely due to the enhanced expression of GLUTs. To investigate the expression profile of these four GLUTs in STYK1/NOK-mediated aerobic glycolysis in the present study, NIH-3T3 cells stably expressing STYK1/NOK were first generated. Cells transfected with pcDNA3.0 or pcDNA3.0-STYK1/NOK were subsequently selected using G418.

Following successful construction, total RNA and proteins were extracted from both the NIH-3T3-pcDNA3.0 and NIH-3T3-pcDNA3.0-STYK1/NOK stable cells. Subsequently, the mRNA and protein expression levels of STYK1/NOK and GLUT1-4 were detected using RT-qPCR (Fig. 1A and B) or western blotting (Fig. 1C), respectively. Fig. 1B shows that the mRNA expression levels of GLUT1-4, particularly GLUT3, were upregulated to varying degrees. Additionally, the results of western blot analysis were consistent with those of RT-qPCR (Fig. 1C). These data indicated that GLUT3 may have a greater impact on cell biological effects driven by STYK1/NOK. In the present study, GLUT3 was selected as the target transporter for subsequent experiments.

GLUT3 silencing reduces STYK1/NOK-mediated aerobic glycolysis. To gain insights into the functional involvement of GLUT3 in STYK1/NOK-induced aerobic glycolysis, siCtrl or siGLUT3 was transiently transfected into NIH-3T3 stable cells. GLUT3 knockdown was assessed by RT-PCR (Fig. 2A), RT-qPCR (Fig. 2B) and western blotting (Fig. 2C). To examine the metabolic influence of the downregulation of GLUT3, the present study subsequently analyzed the glucose uptake 

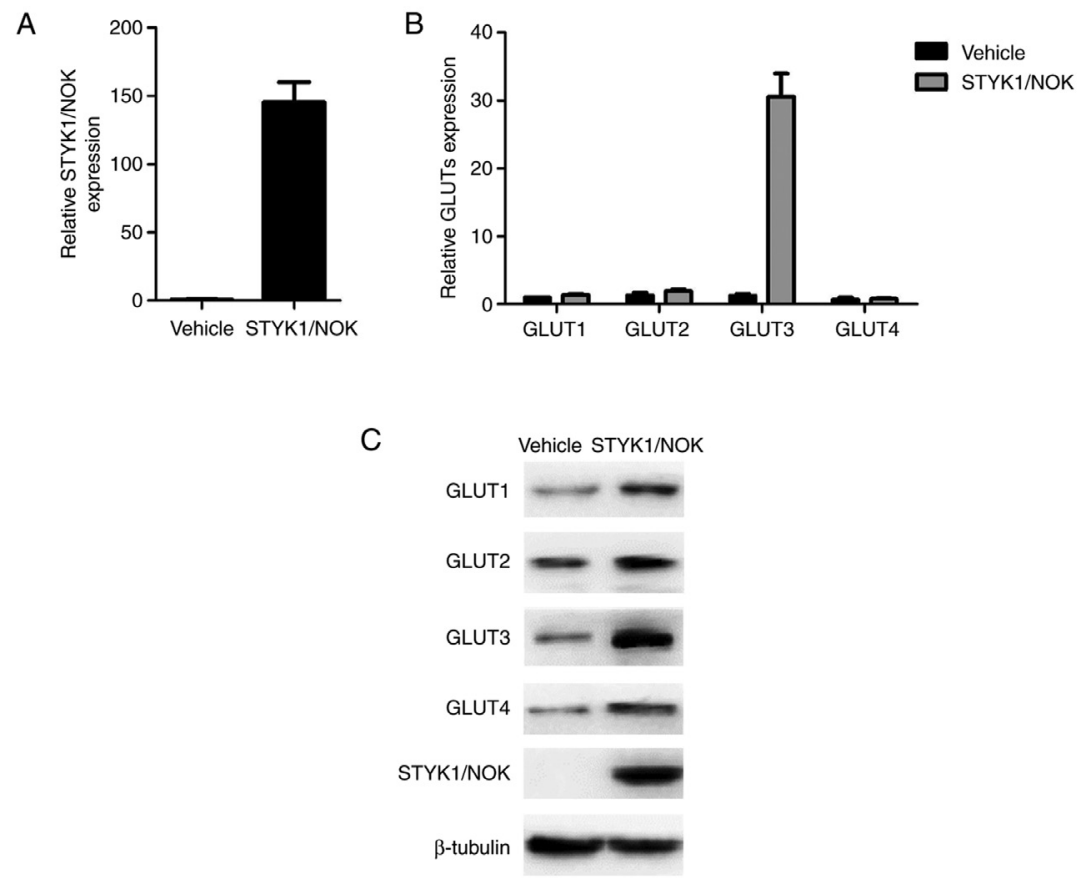

Figure 1. Expression profiles of GLUT1-4 following STYK1/NOK overexpression. Total RNA was extracted from NIH-3T3-pcDNA3.0 (shown as 'vehicle') and NIH-3T3-pcDNA3.0-STYK1/NOK (shown as 'STYK1/NOK') stable cells and subjected to RT-qPCR analysis using primers specific for (A) STYK1/NOK and (B) GLUT1-4. (C) Cell lysates prepared from NIH-3T3 stable cells as described in (A and B) were subjected to western blot analysis. The reaction products were probed using anti-GLUT1, anti-GLUT2, anti-GLUT3, anti-GLUT4 and anti-STYK1/NOK antibodies. $\beta$-tubulin was used as a loading control. GLUT, glucose transporter; STYK1, serine threonine tyrosine kinase 1; NOK, novel oncogene with kinase domain.

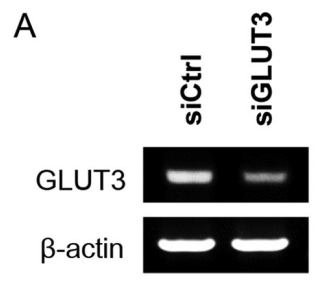

D

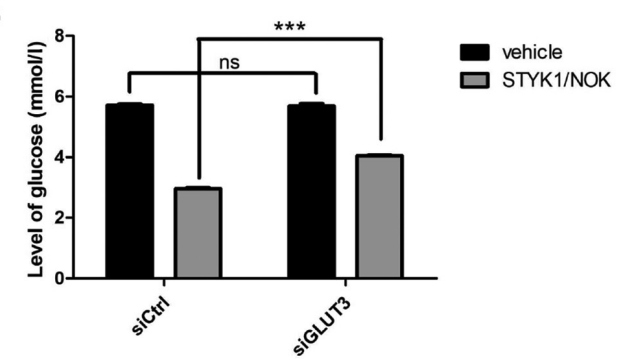

$\mathrm{B}$

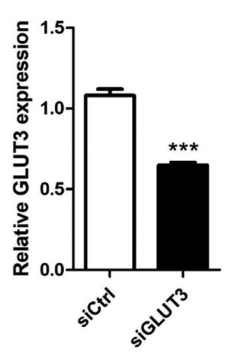

C

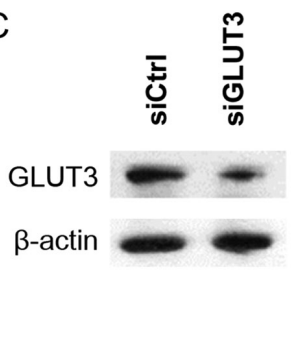

$\mathrm{E}$

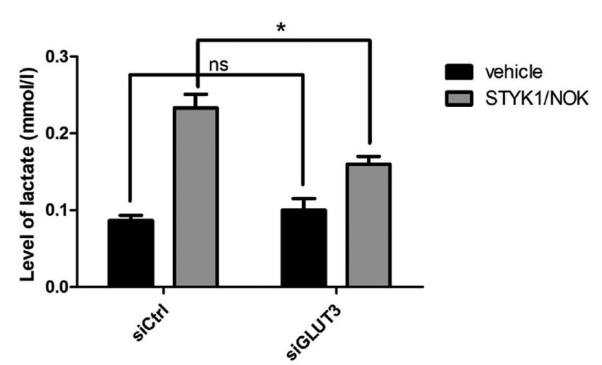

Figure 2. Knockdown of endogenous GLUT3 expression using siGLUT3 reduces STYK1/NOK-driven aerobic glycolysis. An inhibitory effect of siGLUT3 was noted on endogenous GLUT3 expression. NIH-3T3 cells were transiently transfected with either siCtrl or siGLUT3 for 48 h. (A) RT-PCR and (B) RT-qPCR were performed to examine the endogenous mRNA expression levels of GLUT3. (C) Western blot analysis was performed to examine the endogenous protein expression levels of GLUT3. (D) Culture media were harvested from NIH-3T3 stable cells and subjected to detection using a glucose assay kit. (E) NIH-3T3 stable cells were collected and subjected to detection using a lactate assay kit. Each value is presented as the mean \pm SD of three independent experiments in (B, D and E). "P<0.05; ${ }^{* * *} \mathrm{P}<0.001$. ns, no significance; GLUT, glucose transporter; si/siRNA, small interfering RNA; siCtrl, control siRNA; siGLUT3, GLUT3 siRNA; STYK1, serine threonine tyrosine kinase 1; NOK, novel oncogene with kinase domain.

capacity of control and GLUT3-silenced cells by measuring the content of glucose in the culture medium. Notably, although STYK1/NOK overexpression promoted glucose consumption in both groups compared with the vehicle, the increase in STYK1/NOK-mediated glucose uptake was significantly reduced in the siGLUT3 group compared with the siCtrl group (Fig. 2D). Additionally, GLUT3-silenced NIH-3T3-STYK1/NOK stable cells exhibited reduced levels of lactate compared with non-interfering NIH-3T3-STYK1/NOK stable cells (Fig. 2E). Overall, these results suggested that 
A

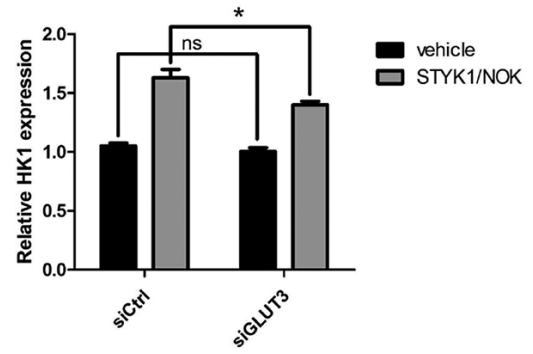

B

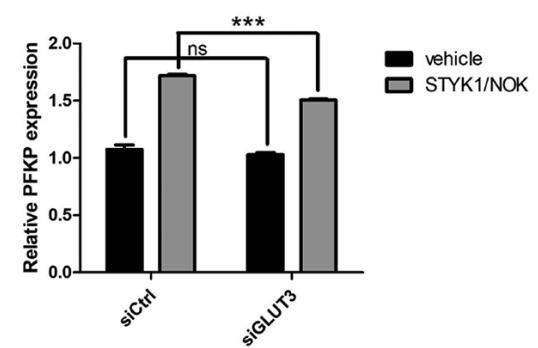

C

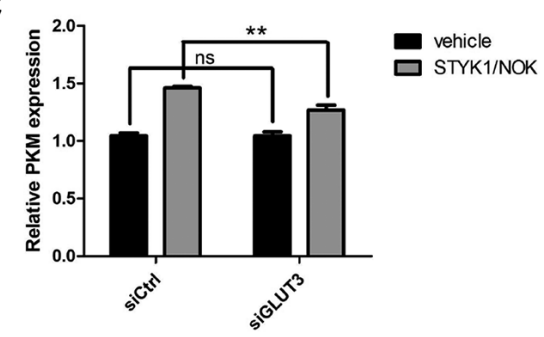

D

siCtrl siGLUT3

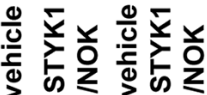

HK1

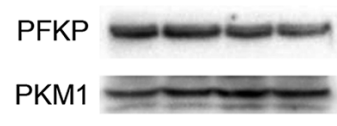

$\beta$-actin
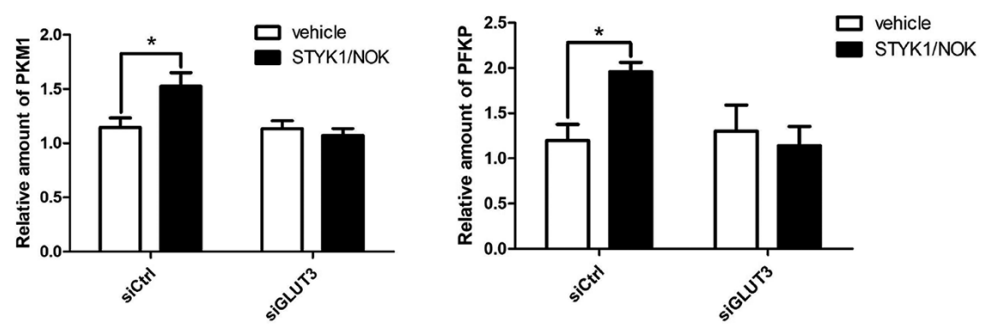

E

siCtrl siGLUT3
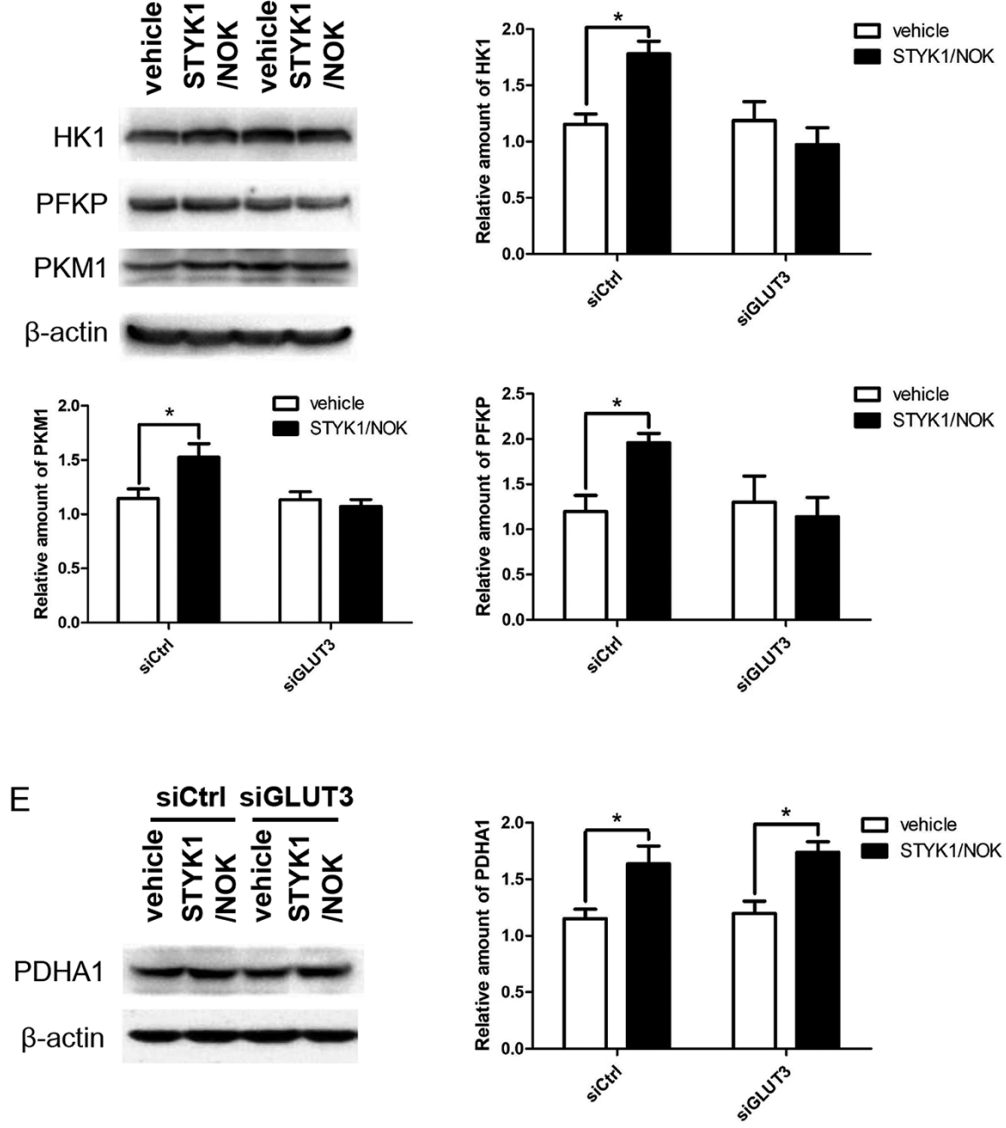

Figure 3. GLUT3 knockdown weakens the effects of STYK1/NOK on key enzymes involved in glycolysis. NIH-3T3 stable cells described as aforementioned were transiently transfected with either siCtrl or siGLUT3 for $48 \mathrm{~h}$. Subsequently, total RNA was extracted and subjected to RT-qPCR analysis using primers specific for (A) HK1, (B) PFKP and (C) PKM. Each value is presented as the mean \pm SD of three independent experiments, which has been adjusted based on $\beta$-actin expression. (D and E) Cell lysates were prepared and subjected to western blot analysis. The reaction products were probed using anti-HK1, anti-PFKP, anti-PKM1 and anti-PDHA1. $\beta$-actin was used as a loading control. The statistical histograms represent the relative amounts of HK1, PFKP, PKM1 and PDHA1, which were quantitated based on the expression levels of $\beta$-actin from three independent assays using the Image $\mathrm{J}$ program. ${ }^{*} \mathrm{P}<0.05$; ${ }^{* *} \mathrm{P}<0.01$; ${ }^{* * * *} \mathrm{P}<0.001$. ns, no significance; GLUT, glucose transporter; STYK1, serine threonine tyrosine kinase 1; NOK, novel oncogene with kinase domain; si/siRNA, small interfering RNA; siCtrl, control siRNA; siGLUT3, GLUT3 siRNA; HK1, hexokinase 1; PFKP, platelet phosphofructokinase; PKM, pyruvate kinase; PDHA1, pyruvate dehydrogenase $\alpha 1$; STYK1, serine threonine tyrosine kinase 1; NOK, novel oncogene with kinase domain.

metabolic reprogramming induced by STYK1/NOK in NIH-3T3 cells was impaired as a consequence of GLUT3 downregulation.

GLUT3 knockdown weakens the effects of STYK1/NOK on the expression levels of key glycolytic enzymes. For further verification, the present study analyzed the expression levels of three rate-limiting enzymes, HK, PFKP and PKM, in the glycolysis signaling pathway, as well as PDHA1, which is one of the subunits of the pyruvate dehydrogenase complex (PDC), which catalyzes the conversion of pyruvate to acetyl-CoA (24). Fig. 3A-C shows that the mRNA expression levels of the three glycolytic enzymes were all upregulated whether or not endogenous GLUT3 was knocked down, yet the degrees of upregulation of glycolytic enzymes in NIH-3T3-STYK1/NOK stable cells were significantly decreased after GLUT3 silencing. Western blot analysis revealed similar results, with the exception that the enhanced expression levels of glycolytic enzymes mediated by STYK1/NOK almost disappeared following GLUT3 knockdown (Fig. 3D).

In contrast to the clear changes in the expression levels of glycolytic enzymes, no marked difference in PDHA1 upregulation mediated by STYK1/NOK was identified between the siCtrl and siGLUT3 groups (Fig. 3E), suggesting that GLUT3 
$24 \mathrm{~h}$

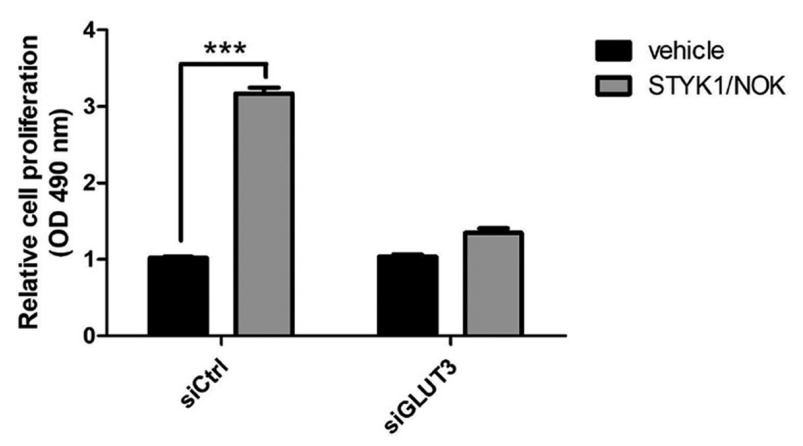

$48 \mathrm{~h}$

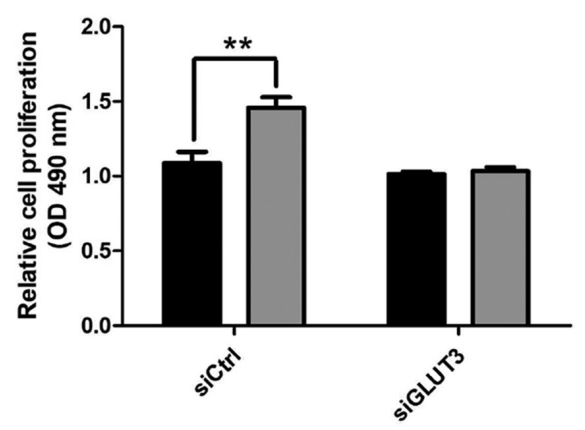

$72 \mathrm{~h}$

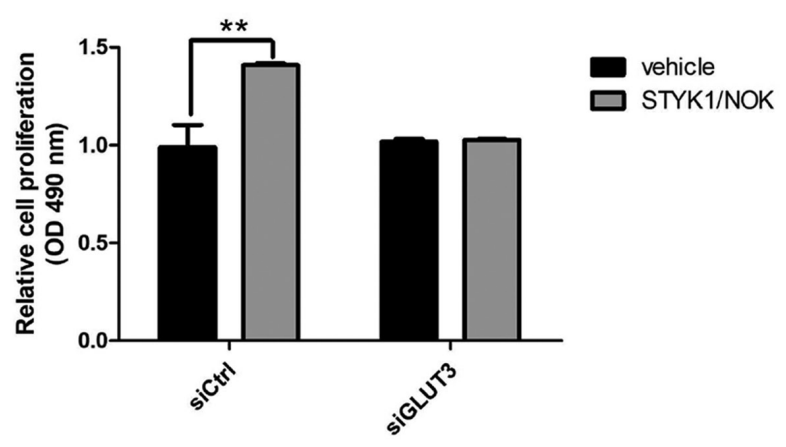

Figure 4. Downregulation of GLUT3 diminishes STYK1/NOK-mediated cell proliferation. NIH-3T3 stable cells described as aforementioned were transiently transfected with either siCtrl or siGLUT3 for 24,48 and $72 \mathrm{~h}$. Subsequently, cells were collected and assayed using MTT. The reaction products were measured at $490 \mathrm{~nm}$ on a plate reader. Each value is presented as the mean $\pm \mathrm{SD}$ of three independent experiments. ${ }^{* *} \mathrm{P}<0.01 ;{ }^{* * *} \mathrm{P}<0.001$. GLUT, glucose transporter; si/siRNA, small interfering RNA; siCtrl, control siRNA; siGLUT3, GLUT3 siRNA; STYK1, serine threonine tyrosine kinase 1 ; NOK, novel oncogene with kinase domain.

may mainly affect STYK1/NOK-controlled aerobic glycolysis, but not the mitochondrial tricarboxylic acid (TCA) cycle.

Loss of GLUT3 diminishes cell proliferation and migration driven by STYK1/NOK. Changes in the glucose metabolism pattern may affect cell proliferation. To investigate the influence of GLUT3 downregulation on NIH-3T3 cell properties, the present study analyzed the proliferation rate of GLUT3-silenced and control NIH-3T3 stable cells using an MTT assay. Fig. 4
A
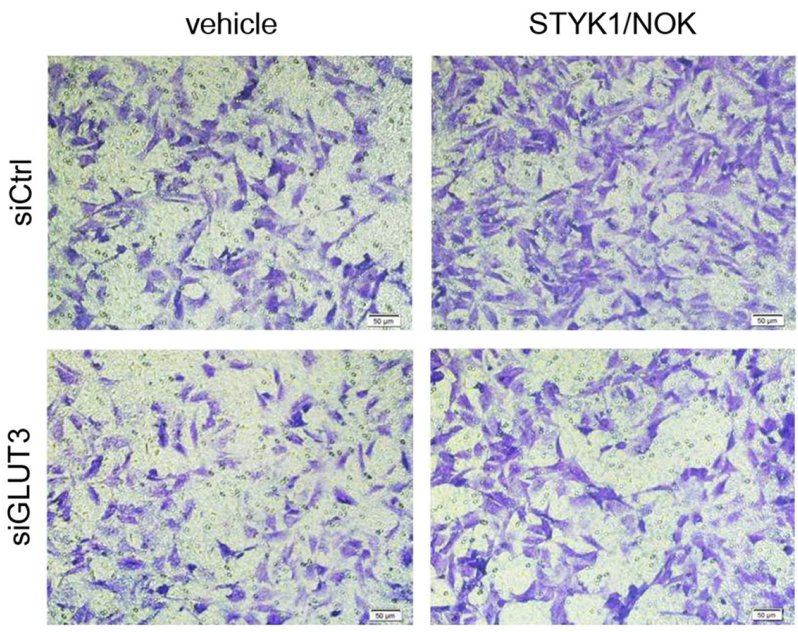

B

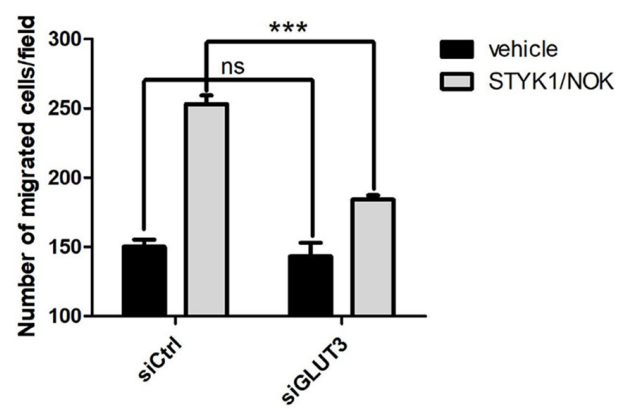

Figure 5. Downregulation of GLUT3 inhibits STYK1/NOK-mediated cell migration. (A) NIH-3T3 stable cells were cultured under different treatment conditions for the Transwell migration assay. A light microscope was used to count the number of stained cells in random fields within each Transwell insert after $24 \mathrm{~h}$ (scale bar, $50 \mu \mathrm{m}$ ). (B) Quantitative analysis of cell migration. Each value is presented as the mean \pm SD of three independent experiments. Following statistical analysis, results were considered to be significant if ${ }^{* * *} \mathrm{P}<0.001$. ns, no significance; GLUT, glucose transporter; siCtrl, control siRNA; siGLUT3, GLUT3 siRNA; STYK1, serine threonine tyrosine kinase 1; NOK, novel oncogene with kinase domain.

shows that STYK1/NOK overexpression markedly promoted cell proliferation in the siCtrl group at several different time points (24, 48 and $72 \mathrm{~h}$ ), which was in agreement with previous studies. However, this increase in cell proliferation caused by STYK1/NOK was markedly reduced following GLUT3 knockdown.

The malignant proliferation of cells is often accompanied by an enhanced migratory ability. The present study subsequently investigated whether loss of GLUT3 might also influence the augmented cell migration caused by STYK1/NOK. A Transwell chamber-based cell migration assay was performed using NIH-3T3 stable cells. As shown in Fig. 5, GLUT3 knockdown diminished NIH-3T3-STYK1/NOK stable cell migration compared with that in the control group. Collectively, these findings revealed that GLUT3 may be crucial to STYK1/NOK-mediated malignant transformation and tumorigenesis.

\section{Discussion}

Aerobic glycolysis, also referred to as the Warburg effect, is a highly conserved phenomenon observed in cancer cells and 
rapidly proliferating cells. According to Warburg's hypothesis, cells preferentially transform glucose to lactate even in the presence of sufficient oxygen (25-27). In cancer cells, this phenotype is mainly driven by the activation of oncogenes or loss of tumor suppressor genes (28). For instance, the Warburg effect can be induced by oncogenic RPTKs, in which a constitutively active form of RPTKs is usually required $(29,30)$. STYK1/NOK represents a typical example of RPTKs, which directly accelerates aerobic glycolysis.

As a consequence of this metabolic reprogramming, cells depend on a high glucose uptake to produce sufficient amounts of ATP to maintain their elevated proliferation rate. Therefore, cancer cells or rapidly proliferating cells exhibit high levels of GLUTs. Among these, class I GLUTs (GLUT1-4) are considered to serve a prominent role in this process due to their ubiquitous expression (10), whereas the involvement of class I GLUTs in the STYK1/NOK-induced Warburg effect remains largely unexplored. To the best of our knowledge, the present study was the first to describe the importance of the GLUT3 transporter in glucose metabolism and cell biological activities in STYK1/NOK-overexpressing NIH-3T3 cells. The data revealed that GLUT3 knockdown led to an impaired capability of basal glucose uptake and lactate production, demonstrating that STYK1/NOK-induced aerobic glycolysis depended on the involvement of GLUT3 to a certain extent. Notably, no apparent effect of GLUT3 downregulation on PDHA1, which is one of the subunits of PDC that catalyzes the conversion of pyruvate to acetyl-CoA, was observed in the present study. This suggested that the influence of GLUT3 on glucose metabolism in STYK1/NOK-overexpressing cells is mainly located upstream of the metabolic pathway, whereas the TCA cycle in mitochondria may not be limited by the downregulation of GLUT3. Our previous study revealed that STYK1/NOK not only markedly enhanced aerobic glycolysis, but also markedly inhibited the process of electron transport and OXPHOS in mitochondria (9). Based on this, a profound study on the mitochondrial function, including the TCA cycle, electron transport and OXPHOS, will be performed in the future to comprehensively analyze the role of GLUT3 in STYK1/NOK-mediated metabolic reprogramming. Additionally, whether other types of GLUTs serve similar or different roles in the biological events caused by STYK1/NOK should be investigated further.

Abnormal energy metabolism is often coupled with changes in cell biological characteristics. For rapidly proliferating cells or cancer cells, more energy and intermediate products can be obtained by reprogramming the metabolic process to meet their own proliferation requirements. Once this metabolic pattern is disturbed, cell proliferation characteristics may also be affected. The present study demonstrated that knockdown of GLUT3 diminished cell proliferation and migration driven by STYK1/NOK, demonstrating that GLUT3 may be crucial to STYK1/NOK-mediated malignant transformation and tumorigenesis. Notably, a considerable number of studies have suggested that STYK1/NOK can activate various proliferation-related signaling pathways or molecules (31-33). A number of the signaling pathways have been reported to strictly regulate the expression and subcellular distribution of GLUTs, for instance, PI3K/Akt/mTOR, hypoxia-inducible factor-1, c-myc and tumor suppressor protein p53 (19,34-36), which provides clues for the further exploration of the upstream molecular mechanism of GLUT3 in STYK1/NOK-overexpressing cells. This is the field of research that will be the focus of our work and studies in the future.

Overall, the data presented in the current study support the notion that tumor cells are highly dependent on GLUTs. To the best of our knowledge, the present study was the first to reveal that GLUT3 downregulation impaired STYK1/NOK-induced metabolic reprogramming, cell proliferation and migration. Therefore, the present study provided insights clarifying the modulatory effects of STYK1/NOK in cell energy metabolism, and for the future development of pharmacological approaches aimed at targeting GLUT3.

\section{Acknowledgements}

Not applicable.

\section{Funding}

The present study was supported by the Natural Science Foundation of Hebei Province (grant no. H2019208216), the Scientific Research Foundation for PhD (grant no. 81/1181286) and the Natural Science Foundation of Hebei Province (grant no. H2020208002).

\section{Availability of data and materials}

All data generated or analyzed during this study are included in this published article.

\section{Authors' contributions}

WS was involved in the conception and design of the present study, performed the experiments and wrote the manuscript. YF and YW were involved in data analysis and interpretation. WS and YF confirm the authenticity of all the raw data. All authors read and approved the final manuscript.

\section{Ethics approval and consent to participate}

Not applicable.

\section{Patient consent for publication}

Not applicable.

\section{Competing interests}

The authors declare that they have no competing interests.

\section{References}

1. Ye X, Ji C, Huang Q, Cheng C, Tang R, Xu J, Zeng L, Dai J, $\mathrm{Wu} \mathrm{Q}, \mathrm{Gu} \mathrm{S}$, et al: Isolation and characterization of a human putative receptor protein kinase cDNA STYK1. Mol Biol Rep 30: 91-96, 2003.

2. Liu L, Yu XZ, Li TS, Song LX, Chen PL, Suo TL, Li YH, Wang SD, Chen Y, Ren YM, et al: A novel protein tyrosine kinase NOK that shares homology with platelet-derived growth factor/fibroblast growth factor receptors induces tumorigenesis and metastasis in nude mice. Cancer Res 64: 3491-3499, 2004. 
3. Cao Q, Chen M, Li Z, Huang W, Jin Y, Ye X and Tong M: High novel oncogene with kinasedomain (NOK) gene expression is associated with the progression of renal cell carcinoma. Clin Lab 62: 179-186, 2016.

4. Chen P, Li WM, Lu Q, Wang J, Yan XL, Zhang ZP and Li XF: Clinicopathologic features and prognostic implications of NOK/STYK1 protein expression in non-small cell lung cancer. BMC Cancer 14: 402, 2014.

5. Jackson KA, Oprea G, Handy J and Kimbro KS: Aberrant STYK1 expression in ovarian cancer tissues and cell lines. J Ovarian Res 2: 15, 2009.

6. Moriai R, Kobayashi D, Amachika T, Tsuji N and Watanabe N: Diagnostic relevance of overexpressed NOK mRNA in breast cancer. Anticancer Res 26 (6c): 4969-4973, 2006.

7. Orang AV, Safaralizadeh R, Hosseinpour Feizi MA and Somi MH: Diagnostic relevance of overexpressed serine threonine tyrosine kinase/novel oncogene with kinase domain (STYK1/NOK) mRNA in colorectal cancer. Asian Pac J Cancer Prev 15: 6685-6689, 2014.

8. Chung S, Tamura K, Furihata M, Uemura M, Daigo Y, Nasu Y, Miki T, Shuin T, Fujioka T, Nakamura $Y$ and Nakagawa $H$ : Overexpression of the potential kinase serine/threonine/tyrosine kinase 1 (STYK 1) in castrationresistant prostate cancer. Cancer Sci 100: 2109-2114, 2009.

9. Shi WY, Yang X, Huang B, Shen WH and Liu L: NOK mediates glycolysis and nuclear PDC associated histone acetylation. Front Biosci (Landmark Ed) 22: 1792-1804, 2017.

10. Thorens B and Mueckler M: Glucose transporters in the 21st Century. Am J Physiol Endocrinol Metab 298: E141-E145, 2010.

11. Barrona CC, Bilan PJ, Tsakiridis T and Tsiani E: Facilitative glucose transporters: Implications for cancer detection, prognosis and treatment. Metabolism 65: 124-139, 2016.

12. Ganapathy V, Thangaraju M and Prasad PD: Nutrient transporters in cancer: Relevance to Warburg hypothesis and beyond. Pharmacol Ther 121: 29-40, 2009.

13. Zhao FQ and Keating AF: Functional properties and genomics of glucose transporters. Curr Genomics 8: 113-128, 2007.

14. Shi Y, Liu S, Ahmad S and Gao Q: Targeting key Transporters in tumor glycolysis as a novel anticancer strategy. Curr Top Med Chem 18: 454-466, 2018.

15. Zhao $\mathrm{M}$ and Zhang Z: Glucose transporter regulation in cancer: A profile and the loops. Crit Rev Eukaryot Gene Expr 26 223-238, 2016.

16. Alexandra $\mathrm{C}$ and $\mathrm{Al}-\mathrm{Hasani} \mathrm{H}$ : Glucose transporters in adipose tissue, liver, and skeletal muscle in metabolic health and disease. Pflugers Arch 472: 1273-1298, 2020.

17. Reckzeh ES and Waldmann H: Development of glucose transporter (GLUT) inhibitors. European J Org Chem 2020: 2321-2329, 2020

18. Groves AM, Shastry M, Rodriguez-Justo M, Malhotra A Endozo R, Davidson T, Kelleher T, Miles KA, Ell PJ and Keshtgar MR: ${ }^{18}$ F-FDG PET and biomarkers for tumour angiogenesis in early breast cancer. Eur J Nucl Med Mol Imaging 38 : 46-52, 2011.

19. Makinoshima H, Takita M, Saruwatari K, Umemura S, Obata Y, Ishii G, Matsumoto S, Sugiyama E, Ochiai A, Abe R, et al: Signaling through the phosphatidylinositol 3-Kinase $(\mathrm{PI} 3 \mathrm{~K}) / \mathrm{mammalian}$ target of rapamycin (mTOR) axis is responsible for aerobic glycolysis mediated by glucose transporter in epidermal growth factor receptor (EGFR)-mutated lung adenocarcinoma. J Biol Chem 290: 17495-17504, 2015.

20. Liu H, Ertay A, Peng P, Li J, Liu D, Xiong H, Zou Y, Qiu H, Hancock D, Yuan X, et al: SGLT1 is required for the survival of triple-negative breast cancer cells via potentiation of EGFR activity. Mol Oncol 13: 1874-1886, 2019.
21. Whiteman EL, Chen JJ and Birnbaum MJ: Platelet-derived growth factor (PDGF) stimulates glucose transport in 3T3-L1 adipocytes overexpressing PDGF receptor by a pathway independent of insulin receptor substrates. Endocrinology 144 3811-3820, 2003.

22. Livak KJ and Schmittgen TD: Analysis of relative gene expression data using real-time quantitative PCR and the 2(-Delta Delta C(T)) method. Methods 25: 402-408, 2001.

23. Zhuo Z, Hu J, Yang X, Chen M, Lei X, Deng L, Yao N, Peng Q, Chen Z, Ye W and Zhang D: Ailanthone inhibits Huh7 cancer cell growth via cell cycle arrest and apoptosis in vitro and in vivo. Sci Rep 5: 16185, 2015.

24. Bose S and Le A: Glucose metabolism in cancer. Adv Exp Med Biol 1063: 3-12, 2018.

25. Warburg O: On respiratory impairment in cancer cells Science 124: 269-270, 1956.

26. Warburg O: On the origin of cancer cells. Science 123: 309-314, 1956.

27. Warburg $\mathrm{O}$ and Minami S: Versuche an uberlebendem carcinom-gewebe. Klin Wochenschr 2: 776-777, 1923.

28. Levine AJ and Puzio-Kuter AM: The control of the metabolic switch in cancers by oncogenes and tumor suppressor genes. Science 330: 1340-1344, 2010.

29. Hitosugi T, Kang S, Vander Heiden MG, Chung TW, Elf S, Lythgoe K, Dong S, Lonial S, Wang X, Chen GZ, et al: Tyrosine phosphorylation inhibits PKM2 to promote the Warburg effect and tumor growth. Sci Signal 2: ra73, 2009.

30. Ji H, Lee JH, Wang Y, Pang Y, Zhang T, Xia Y, Zhong L, Lyu J and Lu Z: EGFR phosphorylates FAM129B to promote Ras activation. Proc Natl Acad Sci USA 113: 644-649, 2016

31. Li YH, Wang YY, Zhong S, Rong ZL, Ren YM, Li ZY, Zhang SP, Chang ZJ and Liu L: Transmembrane helix of novel oncogene with kinase-domain (NOK) influences its oligomerization and limits the activation of RAS/MAPK signaling. Mol Cells 27: 39-45, 2009.

32. Li J, Wu F, Sheng F, Li YJ, Jin D, Ding $X$ and Zhang S NOK/STYK1 interacts with GSK-3 $\beta$ and mediates Ser9 phosphorylation through activated Akt. FEBS Lett 586: 3787-3792, 2012.

33. Huang Z, Ma N, Xiong YL, Wang L, Li WM, Lai YY, Zhang CX, Zhang ZP, Li XF and Zhao JB: Aberrantly high expression of NOK/STYK1 is tightly associated with the activation of the AKT/GSK3 $\beta / \mathrm{N}$-cadherin pathway in non-small cell lung cancer. Onco Targets Ther 12: 10299-10309, 2019.

34. Song K, Li M, Xu XJ, Xuan L, Huang GN, Song XL and Liu QF: HIF-1 $\alpha$ and GLUT1 gene expression is associated with chemoresistance of acute myeloid leukemia. Asian Pac J Cancer Prev 15: 1823-1829, 2014.

35. Miller DM, Thomas SD, Islam A, Muench D and Sedoris K: c-Myc and cancer metabolism. Clin Cancer Res 18: 5546-5553, 2012.

36. Ramos H, Calheiros J, Almeida J, Barcherini V, Santos S, Carvalho ATP, Santos MMM and Saraiva L: SLMP53-1 inhibits tumor cell growth through regulation of glucose metabolism and angiogenesis in a P53-Dependent manner. Int J Mol Sci 21: 596, 2020 .

This work is licensed under a Creative Commons Attribution-NonCommercial-NoDerivatives 4.0 International (CC BY-NC-ND 4.0) License. 